\title{
Laparoscopy versus Laparotomy for the Treatment of BCLC Stages 0 or A Hepatocellular Carcinoma Associated with or without Microvascular Invasion: A Multicenter, Propensity Score Matching Analysis
}

Shi-Ye Yang

Eastern Hepatobiliary Surgery Hospital

Mao-Lin Yan

Fujian Provincial Hospital

Yun-Fei Duan

Changzhou First People's Hospital

Jin-Kai Feng

Eastern Hepatobiliary Surgery Hospital

Jia-Zhou Ye

Guangxi Cancer Hospital and Guangxi Medical University Affiliated Cancer Hospital

Yan-Jun Xiang

Eastern Hepatobiliary Surgery Hospital

Zong-Han Liu

Eastern Hepatobiliary Surgery Hospital

Lei Guo

Eastern Hepatobiliary Surgery Hospital

Jie Xue

Eastern Hepatobiliary Surgery Hospital

Shu-Qun Cheng ( $\sim$ chengshuqun@aliyun.com )

Eastern Hepatobiliary Surgery Hospital https://orcid.org/0000-0001-6760-7470

Wei-Xing Guo

Eastern Hepatobiliary Surgery Hospital

\section{Research Article}

Keywords: hepatocellular carcinoma, microvascular invasion, overall survival, recurrence-free survival, long-term prognosis, propensity score matching, Barcelona Clinic Liver Cancer, laparoscopy, laparotomy, liver resection

Posted Date: February 15th, 2022 
DOI: https://doi.org/10.21203/rs.3.rs-1334237/v1

License: (c) (1) This work is licensed under a Creative Commons Attribution 4.0 International License. Read Full License 


\section{Abstract}

Purpose: To analyze the long-term prognoses of Barcelona Clinic Liver Cancer (BCLC) stages 0 or A hepatocellular carcinoma (HCC) patients associated with or without microvascular invasion (MVI) treated with laparoscopy versus laparotomy using propensity score matched analysis.

Methods: The clinicopathological data of HCC patients at BCLC stages 0 or A from 4 major medical centers were retrospectively reviewed, and sub-grouped in terms of the presence of MVI to explore the effect of MVI on the long-term surgical outcomes. Propensity score matching (PSM) analysis was used to build a matched group of HCC patients for comparison of follow-up indicators between the laparoscopy and laparotomy groups.

Results: $495 \mathrm{HCC}$ patients at BCLC stages 0 or A were enrolled, including 243 in the laparoscopic group and 252 in the laparotomic group. Laparoscopic resection group had a shorter operative time, less blood loss, and lower frequencies of blood transfusion and postoperative complication rates. After PSM, the laparoscopic group had a significantly better overall survival (OS) and recurrence-free survival (RFS) than the laparotomic group. Subgroup analysis demonstrated OS and RFS of patients without MVI were also significantly improved in the laparoscopic group compared with the laparotomic group. However, no significant difference of OS and RFS between two groups was found in patients with MVI after PSM.

Conclusions: Pure laparoscopic hepatectomy for HCC patients at early stages can be performed safely with favorable short-term and long-term outcomes at high-volume liver cancer centers, regardless of the presence of MVI.

\section{Introduction}

Hepatocellular carcinoma (HCC) is one of the most common primary malignancies worldwide. Currently, approximately 740,000 new cases of HCC are diagnosed per year with a five-year survival rate of less than $8.37 \%[1,2]$. Microvascular invasion $(\mathrm{MVI})$, defined as small thrombi consisting of malignant tumor cells in the portal or hepatic venous systems, is an important histopathologic feature of HCC. The presence of $\mathrm{MVI}$ indicates the aggressive behavior of $\mathrm{HCC}$ and predicts bad prognosis and adverse outcomes after hepatectomy or transplantation[3].

To improve the long-term outcomes of HCC patients with MVI, it is reasonable to employ personalized treatment modalities for those patients. A series of studies have shown that surgical resection provides better oncologic outcomes compared with radiofrequency ablation for MVI patients[4-6]. Furthermore, the prognostic value of MVI in different Barcelona Clinic Liver Cancer (BCLC) staging classifications and in eight existing staging systems is various[7, 8]. In addition, anatomic liver resection and wide resection margins are able to provide a better long-term prognosis[9-13]. However, whether different surgical approaches (laparoscopic and open surgery) have a significant impact on the long-term outcome of HCC patients with MVI currently lacks evidence-based research. 
Laparoscopy has unique advantages such as less trauma, less pain, faster postoperative recovery and smaller incisions[14]. Multiple meta-analyses have confirmed the safety and effectiveness of laparoscopic resection applied in HCC patients. Ahmed et al.[15] and Zhou et al.[16] reported that laparoscopic resection is superior to open resection in terms of its perioperative results, and does not compromise the oncological outcomes. It is demonstrated that there was no statistical difference in the radical resection rate between laparoscopic surgery and open resection of $\mathrm{HCC}$ [17], suggesting the emergence of the era of laparoscopic hepatectomy. However, the long-term outcomes of laparoscopy are still unknown in HCC patients with BCLC stages 0 or A. It is unclear whether laparoscopic surgery can bring similar advantages to HCC patients with MVI compared with laparotomy. The operative characteristics and survival rates such as postoperative overall survival (OS) and recurrence-free survival (RFS) in HCC patients with MVI who underwent different surgical methods need to be further explored.

Herein, we aimed to analyze and compare the long-term prognoses of HCC patients associated with or without MVI at BCLC stages 0 or A who were treated with laparoscopy or open hepatectomy using propensity score matching analysis. Besides, MVI was applied to subgroup the HCC patients, with the purpose to investigate the changes of selected indicators in different surgery subgroups.

\section{Methods}

\section{Patient selection}

A total of $1104 \mathrm{HCC}$ patients receiving surgical treatment at four high-volume medical centers (the Eastern Hepatobiliary Surgery Hospital, Fujian Provincial Hospital, Changzhou People's Hospital, and Affiliated Tumor Hospital of Guangxi Medical University) from March 2015 to February 2018 were retrospectively recruited. This study was in accordance with the Declaration of Helsinki (as revised in 2008) and was approved by the Clinical Research Ethics Committee of the four respective institutes. Written informed consent for clinical research was obtained from all the patients prior to enrollment.

The diagnosis of HCC was based on the guidelines for Diagnosis and Treatment of Primary Hepatocellular Carcinoma issued by American Association for the Study of Liver Diseases (AASLD)[18]. Patients who met the criteria of BCLC staging system of 0 or A (ECOG performance status score is 0 , single tumor or up to 3 tumors less than $3 \mathrm{~cm}$, and Child-Pugh class $A-B$ ) were included[19]. The exclusion criteria were as follows: (I) multiple tumors; (II) major vascular (portal vein, hepatic vein or their branches) or bile duct invasion; (III) intrahepatic spread or distant metastatic lesions; (IV) had other concurrent malignant tumors or metastatic liver cancer; $(\mathrm{V})$ be complicated with serious cardiovascular or cerebrovascular diseases; $(\mathrm{VI})$ abnormal coagulation function; and (VII) patients who had the emergency operation due to traumatic or spontaneous tumor rupture.

$\mathrm{MVI}$ is defined as the presence of tumor thrombi within the microscopic vessels adjacent to HCC. The presence of $\mathrm{MVI}$ is related to early tumor recurrence and poor postoperative outcomes after liver resection 
and transplantation[20]. Here, the histopathologic examination was performed to confirm the presence of MVI.

\section{Surgical Procedure}

The laparoscopic hepatectomy was performed as previously reported[21, 22]. Briefly, the patient was in a supine position with full exposure to the location of the tumor. A $10 \mathrm{~mm}$ trocar was placed in the umbilical cord, and laparoscopy was performed to observe the location and size of the liver space-occupying lesion. If the lesion was in the left liver, the main operative site should be located $2 \mathrm{~cm}$ below the costal margin of the midline of the left clavicle. If the lesion was in the right liver, the operative site should be located $2 \mathrm{~cm}$ below the costal margin of the midline of the right clavicle. The ligaments around the liver were separated with an ultrasound knife to make the liver be fully exposed. The liver was prelabeled with an ultrasound knife, and the cutting edge should be more than $2 \mathrm{~cm}$ away from the tumor by LigaSure. The resected tumor specimens were placed in a collection bag. The incision needed hemostasis, and the abdominal cavity drainage tube was placed.

The open resection was conducted traditionally[23]. Briefly, the patient was in a supine position and the scope of resection was depended on tumor size and residual liver volume. General abdominal evaluation was carried out to exclude the metastasis, and liver evaluation was done to determine tumor's location, proximity to vessels, and to exclude multiplicity of tumors by manual palpation and intraoperative ultrasound. Parenchymal transection was performed using Cavitron ultrasonic surgical aspirator, LigaSure and bipolar forceps. Fibrin glue sealant was applied to seal the incision surface of the liver.

\section{Follow-up}

All patients were followed up every two months in the first year after discharge, and every three months thereafter. Follow-up examinations were conducted using laboratory findings (white blood cell count, hemoglobin, platelets count, serum total bilirubin, albumin (ALB), aspartate aminotransferase (AST), glucose, creatinine, alpha-fetoprotein (AFP), prothrombin time (PT)), abdominal ultrasonography, contrast-enhanced computed tomography (CT) or magnetic resonance imaging (MRI). After surgery, the patients were followed up every 3 to 4 months until death or dropout from the follow-up program. A diagnosis of recurrence of HCC was based on CT and/or MRI and elevated serum AFP levels.

\section{Statistical analysis}

Statistical evaluation was performed using SPSS software (IBM SPSS Statistics 21; SPSS Inc., Chicago, IL). Continuous variables with a normal distribution were presented as mean \pm standard deviation (SD); continuous variables with skewed distribution were expressed as median (range). Propensity score matching (PSM) analysis was performed using all the baseline covariates to minimize the selection bias, including age, sex, body mass index (BMI), hepatitis B infection, antiviral treatment, hypertension, 
diabetes, liver cirrhosis, tumor diameter, $\mathrm{MVI}$, resection margin involvement, white blood cell (WBC) count, hemoglobin, platelet count, serum total bilirubin, ALB, AST, glucose, creatinine, AFP and PT.

For the two-group comparison, $t$-test was applied for continuous variables meeting normal distribution, and Mann-Whitney $U$ test was used for continuous variables with skewed distribution. Chi-square test or Fisher's exact test was used for categorical variables as appropriate. The Kaplan-Meier method and logrank test were used for survival analyses.

Univariate and multivariate Cox regression analyses were conducted by COXPH function of survival package from $R$ language (version 3.6.3). Parameters with $P$ values less than 0.05 in the univariate regression analysis were further incorporated into multivariate analysis, and independent prognostic factors were screened out by regressive log-likelihood ratio test. Probability values $(P)<0.05$ were considered to be statistically significant.

\section{Results}

\section{Baseline characteristics}

A total of $1104 \mathrm{HCC}$ patients were collected in this study. Among them, 495 patients (aged from 18 to 70 ) were at BCLC stages 0 or $A, 478$ patients were at stage $B$, and 131 patients were at stage $C$. In this study, $495 \mathrm{HCC}$ patients with BCLC stages 0 or A, including 243 patients who received laparoscopy and 252 who underwent laparotomy, were eventually enrolled and analyzed (Figure 1). After PSM, 189 patients who received laparoscopy and 189 who underwent laparotomy were included. The baseline clinicopathological characteristics of HCC patients before and after PSM are shown in Table 1. After PSM, the statistically significantly different parameters, such as diabetes, tumor diameter, presence of $\mathrm{MVI}$, surgical margin involvement, WBC count, AST and PT, became comparable between the laparoscopic and laparotomic groups (all P凶0.05) (Table 1). 
Table 1

Baseline characteristics of patients before and after PSM

\begin{tabular}{|c|c|c|c|c|c|c|}
\hline \multirow[t]{2}{*}{ Characteristics } & \multicolumn{3}{|l|}{ Before PSM } & \multicolumn{3}{|l|}{ After PSM } \\
\hline & $\begin{array}{l}\text { Laparoscopy } \\
(n=243)\end{array}$ & $\begin{array}{l}\text { laparotomy } \\
(n=252)\end{array}$ & $\begin{array}{l}\mathrm{P}- \\
\text { value }\end{array}$ & $\begin{array}{l}\text { laparoscopy } \\
(n=189)\end{array}$ & $\begin{array}{l}\text { Laparotomy } \\
(n=189)\end{array}$ & $\begin{array}{l}\mathrm{P} \text { - } \\
\text { value }\end{array}$ \\
\hline Age (year) & $\begin{array}{l}59.0 \\
{[50.0 ; 66.0]}\end{array}$ & $\begin{array}{l}60.0 \\
{[51.0 ; 68.0]}\end{array}$ & 0.347 & $\begin{array}{l}59.0 \\
{[50.0 ; 66.0]}\end{array}$ & $\begin{array}{l}59.0 \\
{[50.0 ; 66.0]}\end{array}$ & 0.965 \\
\hline Sex, male (\%) & $202(83.1 \%)$ & $\begin{array}{l}217 \\
(86.1 \%)\end{array}$ & 0.426 & $159(84.1 \%)$ & $162(85.7 \%)$ & 0.774 \\
\hline BMI & $\begin{array}{l}22.9 \\
{[20.9 ; 25.0]}\end{array}$ & $\begin{array}{l}22.3 \\
{[20.3 ; 24.8]}\end{array}$ & 0.144 & $\begin{array}{l}22.8 \\
{[20.9 ; 24.9]}\end{array}$ & $\begin{array}{l}22.5 \\
{[20.3 ; 25.0]}\end{array}$ & 0.524 \\
\hline $\begin{array}{l}\text { Hepatitis B } \\
\text { infection (\%) }\end{array}$ & $188(77.4 \%)$ & $\begin{array}{l}208 \\
(82.5 \%)\end{array}$ & 0.185 & $154(81.5 \%)$ & 157 (83.1\%) & 0.788 \\
\hline Antiviral (\%) & 75 (30.9\%) & $61(24.2 \%)$ & 0.119 & $50(26.5 \%)$ & $50(26.5 \%)$ & 1.000 \\
\hline Hypertension (\%) & $53(21.8 \%)$ & $70(27.8 \%)$ & 0.152 & $44(23.3 \%)$ & $51(27.0 \%)$ & 0.477 \\
\hline Diabetes (\%) & $39(16.0 \%)$ & $61(24.2 \%)$ & 0.032 & 35 (18.5\%) & $44(23.3 \%)$ & 0.312 \\
\hline Liver cirrhosis (\%) & 175 (72.0\%) & $\begin{array}{l}169 \\
(67.1 \%)\end{array}$ & 0.272 & $133(70.4 \%)$ & $132(69.8 \%)$ & 1.000 \\
\hline $\begin{array}{l}\text { Tumor diameter } \\
\text { (cm) }\end{array}$ & & & $<0.001$ & & & 0.536 \\
\hline$\leq 5$ & $141(58.0 \%)$ & $96(38.1 \%)$ & & $90(47.6 \%)$ & $83(43.9 \%)$ & \\
\hline$>5$ & $102(42.0 \%)$ & $\begin{array}{l}156 \\
(61.9 \%)\end{array}$ & & $99(52.4 \%)$ & $106(56.1 \%)$ & \\
\hline MVI & & & 0.024 & & & 0.916 \\
\hline Negative & $132(54.3 \%)$ & $\begin{array}{l}163 \\
(64.7 \%)\end{array}$ & & $115(60.8 \%)$ & $117(61.9 \%)$ & \\
\hline Positive & $111(45.7 \%)$ & $89(35.3 \%)$ & & $74(39.2 \%)$ & $72(38.1 \%)$ & \\
\hline Resection margin & & & 0.017 & & & 1.000 \\
\hline Wide & 95 (39.1\%) & $72(28.6 \%)$ & & $59(31.2 \%)$ & $58(30.7 \%)$ & \\
\hline Narrow & $148(60.9 \%)$ & $\begin{array}{l}180 \\
(71.4 \%)\end{array}$ & & $130(68.8 \%)$ & $131(69.3 \%)$ & \\
\hline $\begin{array}{l}\text { White blood cell } \\
\text { count, } \times 10 \otimes / L\end{array}$ & & & 0.034 & & & 0.179 \\
\hline
\end{tabular}

PSM, propensity score matching; BMI, body mass index; MVI, microvascular invasion; ALB, albumin; AST, aspartate aminotransferase; AFP, alpha-fetoprotein; PT, prothrombin time 


\begin{tabular}{|c|c|c|c|c|c|c|}
\hline \multirow[t]{2}{*}{ Characteristics } & \multicolumn{3}{|l|}{ Before PSM } & \multicolumn{3}{|l|}{ After PSM } \\
\hline & $\begin{array}{l}\text { Laparoscopy } \\
(n=243)\end{array}$ & $\begin{array}{l}\text { laparotomy } \\
(n=252)\end{array}$ & $\begin{array}{l}\mathrm{P}- \\
\text { value }\end{array}$ & $\begin{array}{l}\text { laparoscopy } \\
(n=189)\end{array}$ & $\begin{array}{l}\text { Laparotomy } \\
(\mathrm{n}=189)\end{array}$ & $\begin{array}{l}P \text { - } \\
\text { value }\end{array}$ \\
\hline$\leq 4$ & $23(9.47 \%)$ & $41(16.3 \%)$ & & $21(11.1 \%)$ & $31(16.4 \%)$ & \\
\hline$>4$ & $220(90.5 \%)$ & $\begin{array}{l}211 \\
(83.7 \%)\end{array}$ & & 168 (88.9\%) & $158(83.6 \%)$ & \\
\hline Hemoglobin, g/L, & & & 0.290 & & & 0.620 \\
\hline$\leq 100$ & $7(2.88 \%)$ & $13(5.16 \%)$ & & $7(3.70 \%)$ & 10 (5.29\%) & \\
\hline$>100$ & $236(97.1 \%)$ & $\begin{array}{l}239 \\
(94.8 \%)\end{array}$ & & $182(96.3 \%)$ & $179(94.7 \%)$ & \\
\hline $\begin{array}{l}\text { Platelet count, } \\
\times 10 \rrbracket / L\end{array}$ & & & 0.552 & & & 0.392 \\
\hline$\leq 100$ & 23 (9.47\%) & 29 (11.5\%) & & $16(8.47 \%)$ & $22(11.6 \%)$ & \\
\hline$>100$ & $220(90.5 \%)$ & $\begin{array}{l}223 \\
(88.5 \%)\end{array}$ & & $173(91.5 \%)$ & 167 (88.4\%) & \\
\hline $\begin{array}{l}\text { Serum total } \\
\text { bilirubin, umol/L }\end{array}$ & & & 0.599 & & & 1.000 \\
\hline$\leq 17.5$ & 190 (78.2\%) & $\begin{array}{l}191 \\
(75.8 \%)\end{array}$ & & $146(77.2 \%)$ & $146(77.2 \%)$ & \\
\hline$>17.5$ & $53(21.8 \%)$ & $61(24.2 \%)$ & & $43(22.8 \%)$ & $43(22.8 \%)$ & \\
\hline $\operatorname{ALB}(\mathrm{g} / \mathrm{L})$ & & & 0.893 & & & 1.000 \\
\hline$\leq 35$ & 25 (10.3\%) & $24(9.52 \%)$ & & 17 (8.99\%) & $18(9.52 \%)$ & \\
\hline$>35$ & $218(89.7 \%)$ & $\begin{array}{l}228 \\
(90.5 \%)\end{array}$ & & $172(91.0 \%)$ & $171(90.5 \%)$ & \\
\hline AST (U/L) & & & 0.010 & & & 0.502 \\
\hline$\leq 45$ & $183(75.3 \%)$ & $\begin{array}{l}162 \\
(64.3 \%)\end{array}$ & & 135 (71.4\%) & $128(67.7 \%)$ & \\
\hline$\varangle 45$ & 60 (24.7\%) & $90(35.7 \%)$ & & $54(28.6 \%)$ & $61(32.3 \%)$ & \\
\hline Glucose, $\mathrm{mmol} / \mathrm{L}$ & & & 0.485 & & & 0.321 \\
\hline$\leq 7$ & 218 (89.7\%) & $\begin{array}{l}220 \\
(87.3 \%)\end{array}$ & & $172(91.0 \%)$ & $165(87.3 \%)$ & \\
\hline$>7$ & 25 (10.3\%) & $32(12.7 \%)$ & & 17 (8.99\%) & $24(12.7 \%)$ & \\
\hline
\end{tabular}

PSM, propensity score matching; BMI, body mass index; MVI, microvascular invasion; ALB, albumin; AST, aspartate aminotransferase; AFP, alpha-fetoprotein; PT, prothrombin time 


\begin{tabular}{|c|c|c|c|c|c|c|}
\hline \multirow[t]{2}{*}{ Characteristics } & \multicolumn{3}{|l|}{ Before PSM } & \multicolumn{3}{|l|}{ After PSM } \\
\hline & $\begin{array}{l}\text { Laparoscopy } \\
(n=243)\end{array}$ & $\begin{array}{l}\text { laparotomy } \\
(n=252)\end{array}$ & $\begin{array}{l}\mathrm{P}- \\
\text { value }\end{array}$ & $\begin{array}{l}\text { laparoscopy } \\
(n=189)\end{array}$ & $\begin{array}{l}\text { Laparotomy } \\
(n=189)\end{array}$ & $\begin{array}{l}\mathrm{P} \text { - } \\
\text { value }\end{array}$ \\
\hline Creatinine, umol/L & & & 0.722 & & & 1.000 \\
\hline$\leq 106$ & $236(97.1 \%)$ & $\begin{array}{l}247 \\
(98.0 \%)\end{array}$ & & $184(97.4 \%)$ & 185 (97.9\%) & \\
\hline$>106$ & $7(2.88 \%)$ & $5(1.98 \%)$ & & $5(2.65 \%)$ & $4(2.12 \%)$ & \\
\hline $\operatorname{AFP}(\mathrm{ng} / \mathrm{ml})$ & & & 0.143 & & & 0.587 \\
\hline$\leq 400$ & $174(71.6 \%)$ & $\begin{array}{l}164 \\
(65.1 \%)\end{array}$ & & 128 (67.7\%) & $122(64.6 \%)$ & \\
\hline$>400$ & $69(28.4 \%)$ & $88(34.9 \%)$ & & $61(32.3 \%)$ & $67(35.4 \%)$ & \\
\hline $\mathrm{PT}(\mathrm{s})$ & & & 0.007 & & & 0.622 \\
\hline$\leq 13.5$ & 197 (81.1\%) & $\begin{array}{l}177 \\
(70.2 \%)\end{array}$ & & 149 (78.8\%) & $144(76.2 \%)$ & \\
\hline$>13.5$ & $46(18.9 \%)$ & $75(29.8 \%)$ & & $40(21.2 \%)$ & $45(23.8 \%)$ & \\
\hline
\end{tabular}

\section{Survival analyses in HCC patients treated by laparoscopy or laparotomy before and after PSM}

Before PSM, in the laparoscopic group, the OS at 1, 2, 3 and 5 years were $93.7 \%, 85.9 \%, 82.6 \%$ and $78.8 \%$, respectively, and the RFS at 1, 2, 3 and 5 years were $81 \%, 77.7 \%, 75 \%$ and $71.4 \%$, respectively. As for patients in the laparotomic group, the OS at 1, 2, 3 and 5 years were $88.1 \%, 77.3 \%, 67.9 \%$ and $55.7 \%$, respectively, and the RFS at 1, 2, 3 and 5 years were $72.8 \%, 66.1 \%, 58.7 \%$ and $53.6 \%$, respectively. The results indicated that the OS and RFS were significantly better in the laparoscopic group compared with the laparotomic group (both $\mathrm{P}<0.001$ ) (Figure $2 \mathrm{~A}-\mathrm{B}$ ).

After PSM, in the laparoscopic group, the OS at 1, 2, 3 and 5 years were $93.0 \%, 84.4 \%, 80.3 \%$ and $75.8 \%$, respectively, and the RFS at $1,2,3$ and 5 years were $80.5 \%, 76.9 \%, 73.4 \%$ and $70.9 \%$, respectively. In the laparotomic group, the OS at 1, 2, 3 and 5 years were $89.9 \%, 78.9 \%, 70.9 \%$ and $61 \%$, respectively, and the RFS at $1,2,3$ and 5 years were $73.8 \%, 68.2 \%, 62 \%$ and $56.8 \%$, respectively. The results after PSM still showed a better OS and RFS in the laparoscopic group compared with the laparotomic group (both $\mathrm{P}<0.05$ ) (Figure 2C-D).

Subgroup analyses in HCC patients with MVI treated with laparoscopy or laparotomy before and after PSM 
As shown in Figure 3 , before PSM, both OS and RFS of MVI negative patients were significantly better in the laparoscopic group compared with the laparotomic group (both $\mathrm{P}<0.001$ ) (Figure 3A-B). The OS and RFS of MVI positive patients were also significantly improved in the laparoscopic group compared with the laparotomic group (both $\mathrm{P}<0.05$ ) (Figure $3 \mathrm{C}-\mathrm{D}$ ). After PSM, the OS and RFS of MVI negative patients were also significantly better in the laparoscopic group compared with the laparotomic group (both $\mathrm{P}<0.05$ ) (Figure 4A-B). However, no significant difference for OS and RFS was observed in MVI positive patients in the laparoscopic and laparotomic groups after PSM (Figure 4C-D).

\section{Independent risk factors for OS and RFS in HCC patients before and after PSM}

Before PSM, HBV infection, diabetes, abnormal level of AST and AFP, tumor size $>5 \mathrm{~cm}$, the presence of $\mathrm{MVI}$, and open resection approach were independent predictive factors of OS. As for RFS, multivariate analyses demonstrated that diabetes, abnormal levels of AST, TBIL and AFP, tumor size $>5 \mathrm{~cm}$, the presence of $\mathrm{MVI}$, and open resection approach were the independent risk factors (all $\mathrm{P}<0.05$ ) (Table 2 ).

After PSM, as presented in Table 3, multivariate analyses identified that the history of HBV infection and the absence of antiviral therapy were the independent risk factors of OS. Besides, it was identified that abnormal levels of AST, tumor diameter $5 \mathrm{~cm}, \mathrm{MVI}$ and open resection method were the independent risk factors for both OS and RFS (all $P<0.05$ ). 
Table 3

Univariate and multivariate analysis of overall survival and recurrence-free survival for patients after PSM

\section{Table 2 Univariate and multivariate analysis of OS and RFS for patients before PSM}

\begin{tabular}{|c|c|c|c|c|c|c|c|c|}
\hline \multirow[t]{3}{*}{ Characteristics } & \multicolumn{4}{|l|}{ OS } & \multicolumn{4}{|l|}{ RFS } \\
\hline & \multirow{2}{*}{$\begin{array}{l}\begin{array}{l}\text { Univariate } \\
\text { analysis }\end{array} \\
\text { P-value }\end{array}$} & \multicolumn{3}{|c|}{ Multivariate analysis } & \multirow{2}{*}{ 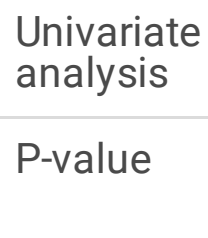 } & \multicolumn{3}{|c|}{ Multivariate analysis } \\
\hline & & $\mathrm{HR}$ & $95 \% \mathrm{Cl}$ & $\begin{array}{l}\text { P- } \\
\text { value }\end{array}$ & & $\mathrm{HR}$ & $95 \% \mathrm{Cl}$ & $\begin{array}{l}\text { P- } \\
\text { value }\end{array}$ \\
\hline Age (year) & 0.700 & & & & 0.700 & 0.71 & $\begin{array}{l}(0.44- \\
1.16)\end{array}$ & 0.174 \\
\hline $\begin{array}{l}\text { Sex (male vs } \\
\text { female) }\end{array}$ & 0.900 & & & & 0.800 & & & \\
\hline HBV & 0.003 & 2.43 & $\begin{array}{l}(1.36- \\
4.35)\end{array}$ & 0.003 & 0.078 & & & \\
\hline
\end{tabular}

\begin{tabular}{|c|c|c|c|c|c|c|c|c|}
\hline $\begin{array}{l}\text { Antiviral (yes vs } \\
\text { no) }\end{array}$ & 0.053 & & & & 0.400 & & & \\
\hline $\begin{array}{l}\text { Hypertension } \\
\text { (yes vs no) }\end{array}$ & 0.044 & & & & 0.110 & & & \\
\hline $\begin{array}{l}\text { Diabetes (yes vs } \\
\text { no) }\end{array}$ & 0.010 & 1.66 & $\begin{array}{l}(1.15- \\
2.41)\end{array}$ & 0.007 & 0.021 & 1.55 & $\begin{array}{l}(1.10- \\
2.18)\end{array}$ & 0.013 \\
\hline $\begin{array}{l}\text { WBC }(\leq 4 \text { vs }>4) \\
\left({ }^{*} 10^{9} / \mathrm{L}\right)\end{array}$ & $>0.9$ & & & & 0.600 & & & \\
\hline $\begin{array}{l}\mathrm{HGB}(\leq 100 \mathrm{vs} \\
>100)\left({ }^{*} 10^{9} / \mathrm{L}\right)\end{array}$ & 0.084 & & & & 0.400 & & & \\
\hline $\begin{array}{l}\mathrm{PT}(\leq 13.5 \text { vs } \\
>13.5)(\mathrm{sec})\end{array}$ & 0.026 & & & & 0.100 & & & \\
\hline $\begin{array}{l}\mathrm{PLT}(\leq 100 \text { vs } \\
>100)\left({ }^{*} 10^{9} / \mathrm{L}\right)\end{array}$ & $>0.9$ & & & & 0.500 & & & \\
\hline $\begin{array}{l}\operatorname{ALB}(\leq 35 \text { vs }>35) \\
\left(* 10^{9} / \mathrm{L}\right)\end{array}$ & 0.010 & & & & 0.130 & & & \\
\hline $\begin{array}{l}\text { AST ( } \leq 45 \text { vs } \\
>45)\left({ }^{*} 10^{9} / \mathrm{L}\right)\end{array}$ & $<0.001$ & 1.57 & $\begin{array}{l}(1.10- \\
2.25)\end{array}$ & 0.013 & $<0.001$ & 1.54 & $\begin{array}{l}(1.11- \\
2.13)\end{array}$ & 0.010 \\
\hline $\begin{array}{l}\text { Glu }(\leq 7 \text { vs }>7) \\
\left({ }^{\star} 10^{9} / \mathrm{L}\right)\end{array}$ & 0.130 & & & & 0.400 & & & \\
\hline $\begin{array}{l}\operatorname{Cr}(\leq 106 \text { vs } \\
>106)\left({ }^{*} 10^{9} / \mathrm{L}\right)\end{array}$ & $>0.9$ & & & & 0.300 & & & \\
\hline
\end{tabular}




\begin{tabular}{|c|c|c|c|c|c|c|c|c|}
\hline $\begin{array}{l}\text { TBIL ( } \leq 17.5 \mathrm{vs} \\
>17.5)(\text { umol/L) }\end{array}$ & 0.049 & 1.40 & $\begin{array}{l}(0.97- \\
2.03)\end{array}$ & 0.074 & 0.027 & 1.44 & $\begin{array}{l}(1.03- \\
2.01)\end{array}$ & 0.033 \\
\hline $\begin{array}{l}\text { Cirrhosis (yes vs } \\
\text { no) }\end{array}$ & 0.900 & & & & 0.600 & & & \\
\hline $\begin{array}{l}\text { AFP ( } \leq 400 \mathrm{vs} \\
>400)(\mathrm{umol} / \mathrm{L})\end{array}$ & $<0.001$ & 1.53 & $\begin{array}{l}(1.08- \\
2.17)\end{array}$ & 0.016 & $<0.001$ & 1.42 & $\begin{array}{l}(1.03- \\
1.95)\end{array}$ & 0.031 \\
\hline $\begin{array}{l}\text { Tumor }(>5 \text { vs } \leq 5) \\
(\mathrm{cm})\end{array}$ & $<0.001$ & 2.44 & $\begin{array}{l}(1.63- \\
3.65)\end{array}$ & $<0.001$ & $<0.001$ & 1.84 & $\begin{array}{l}(1.31- \\
2.60)\end{array}$ & $<0.001$ \\
\hline $\begin{array}{l}\text { Margin (>1 vs } \\
\leq 1)(\mathrm{cm})\end{array}$ & 0.500 & & & & 0.500 & & & \\
\hline $\begin{array}{l}\text { MVI (positive vs } \\
\text { negative) }\end{array}$ & 0.019 & 1.46 & $\begin{array}{l}(1.02- \\
2.10)\end{array}$ & 0.041 & 0.003 & 1.41 & $\begin{array}{l}(1.03- \\
1.95)\end{array}$ & 0.034 \\
\hline $\begin{array}{l}\text { Operative type } \\
\text { (lap vs open) }\end{array}$ & $<0.001$ & 1.58 & $\begin{array}{l}(1.08- \\
2.30)\end{array}$ & 0.018 & $<0.001$ & 1.46 & $\begin{array}{l}(1.06- \\
2.02)\end{array}$ & 0.021 \\
\hline \multicolumn{9}{|c|}{$\begin{array}{l}\text { PSM, propensity score matching; OS, overall survival; RFS, recurrent-free survival; } \mathrm{Cl} \text {, confidence } \\
\text { interval; HBV, hepatitis B virus; WBC, white blood cells; HGB, hemoglobin; PT, prothrombin time; PLT, } \\
\text { platelets; ALB, albumin; AST, aspartate aminotransferase; Glu, glucose; Cr, creatinine; TBIL, serum total } \\
\text { bilirubin; AFP, alpha-fetoprotein; MVI, microvascular invasion }\end{array}$} \\
\hline
\end{tabular}




\begin{tabular}{|c|c|c|c|c|c|c|c|c|}
\hline \multirow[t]{3}{*}{ Characteristics } & \multicolumn{4}{|l|}{ os } & \multicolumn{4}{|l|}{ RFS } \\
\hline & \multirow{2}{*}{$\begin{array}{l}\begin{array}{l}\text { Univariate } \\
\text { analysis }\end{array} \\
\text { P-value }\end{array}$} & \multicolumn{3}{|c|}{ Multivariate analysis } & \multirow{2}{*}{$\begin{array}{l}\begin{array}{l}\text { Univariate } \\
\text { analysis }\end{array} \\
\text { P-value }\end{array}$} & \multicolumn{3}{|c|}{ Multivariate analysis } \\
\hline & & HR & $95 \% \mathrm{Cl}$ & $\begin{array}{l}\mathrm{P}- \\
\text { value }\end{array}$ & & HR & $95 \% \mathrm{Cl}$ & $\begin{array}{l}P- \\
\text { value }\end{array}$ \\
\hline Age (year) & $>0.9$ & & & & $>0.9$ & & & \\
\hline $\begin{array}{l}\text { Sex (male vs } \\
\text { female) }\end{array}$ & 0.600 & & & & 0.200 & & & \\
\hline HBV & 0.011 & 2.25 & $\begin{array}{l}(1.25- \\
4.07)\end{array}$ & 0.007 & 0.300 & & & \\
\hline $\begin{array}{l}\text { Antiviral (yes vs } \\
\text { no) }\end{array}$ & 0.010 & 1.53 & $\begin{array}{l}(1.05- \\
2.22)\end{array}$ & 0.026 & 0.200 & & & \\
\hline $\begin{array}{l}\text { Hypertension } \\
\text { (yes vs no) }\end{array}$ & 0.200 & & & & 0.200 & & & \\
\hline $\begin{array}{l}\text { Diabetes (yes vs } \\
\text { no) }\end{array}$ & 0.094 & & & & 0.110 & & & \\
\hline $\begin{array}{l}\text { WBC }(\leq 4 \text { vs }>4) \\
\left({ }^{*} 10^{9 / L}\right)\end{array}$ & 0.800 & & & & 0.500 & & & \\
\hline $\begin{array}{l}\text { HGB }(\leq 100 \text { vs } \\
>100)\left({ }^{*} 10^{9} / L\right)\end{array}$ & 0.200 & & & & 0.700 & & & \\
\hline $\begin{array}{l}\mathrm{PT}(\leq 13.5 \mathrm{vs} \\
>13.5)(\mathrm{sec})\end{array}$ & 0.500 & & & & 0.800 & & & \\
\hline $\begin{array}{l}\text { PLT ( } \leq 100 \text { vs } \\
>100)\left({ }^{*} 10^{9} / \mathrm{L}\right)\end{array}$ & 0.800 & & & & 0.800 & & & \\
\hline $\begin{array}{l}\text { ALB }(\leq 35 \text { vs }>35) \\
\left({ }^{*} 10^{9} / \mathrm{L}\right)\end{array}$ & 0.011 & & & & 0.032 & & & \\
\hline $\begin{array}{l}\text { AST ( } \leq 45 \text { vs } \\
>45)\left({ }^{*} 10^{9} / \mathrm{L}\right)\end{array}$ & $<0.001$ & 1.70 & $\begin{array}{l}(1.20- \\
2.42)\end{array}$ & 0.003 & $<0.001$ & 1.61 & $\begin{array}{l}(1.17- \\
2.22)\end{array}$ & 0.004 \\
\hline $\begin{array}{l}\text { Glu }(\leq 7 \text { vs }>7) \\
\left({ }^{*} 10^{9} / \mathrm{L}\right)\end{array}$ & 0.200 & & & & 0.400 & & & \\
\hline $\begin{array}{l}\operatorname{Cr}\left(\leq 10^{6} \text { vs }>10^{6}\right) \\
\left({ }^{\star} 10^{9} / \mathrm{L}\right)\end{array}$ & $>0.9$ & & & & 0.600 & & & \\
\hline
\end{tabular}

PSM, propensity score matching; OS, overall survival; RFS, recurrent-free survival; $\mathrm{Cl}$, confidence interval; HBV, hepatitis B virus; WBC, white blood cells; HGB, hemoglobin; PT, prothrombin time; PLT, platelets; ALB, albumin; AST, aspartate aminotransferase; Glu, glucose; $\mathrm{Cr}$, creatinine; TBIL, serum total bilirubin; AFP, alpha-fetoprotein; MVI, microvascular invasion 


\begin{tabular}{|c|c|c|c|c|c|c|c|c|}
\hline \multirow[t]{3}{*}{ Characteristics } & \multicolumn{4}{|l|}{ os } & \multicolumn{4}{|l|}{ RFS } \\
\hline & \multirow{2}{*}{$\begin{array}{l}\text { Univariate } \\
\text { analysis } \\
\text { P-value }\end{array}$} & \multicolumn{3}{|c|}{ Multivariate analysis } & \multirow{2}{*}{$\begin{array}{l}\text { Univariate } \\
\text { analysis } \\
\text { P-value }\end{array}$} & \multicolumn{3}{|c|}{ Multivariate analysis } \\
\hline & & HR & $95 \% \mathrm{Cl}$ & $\begin{array}{l}\mathrm{P}- \\
\text { value }\end{array}$ & & HR & $95 \% \mathrm{Cl}$ & $\begin{array}{l}\mathrm{P}- \\
\text { value }\end{array}$ \\
\hline $\begin{array}{l}\text { TBIL ( } \leq 17.5 \text { vs } \\
>17.5) \text { (umol/L) }\end{array}$ & 0.200 & & & & 0.200 & & & \\
\hline $\begin{array}{l}\text { Cirrhosis (yes vs } \\
\text { no) }\end{array}$ & 0.700 & & & & 0.500 & & & \\
\hline $\begin{array}{l}\operatorname{AFP}(\leq 400 \text { vs } \\
>400)(\text { umol/L) }\end{array}$ & 0.008 & & & & 0.021 & & & \\
\hline $\begin{array}{l}\text { Tumor }(>5 \text { vs } \leq 5) \\
(\mathrm{cm})\end{array}$ & $<0.001$ & 2.63 & $\begin{array}{l}(1.77- \\
3.91)\end{array}$ & $<0.001$ & $<0.001$ & 1.91 & $\begin{array}{l}(1.37- \\
2.68)\end{array}$ & $<0.001$ \\
\hline $\begin{array}{l}\text { Margin }(>1 \text { vs } \\
\leq 1)(\mathrm{cm})\end{array}$ & 0.700 & & & & 0.600 & & & \\
\hline $\begin{array}{l}\text { MVI (positive vs } \\
\text { negative) }\end{array}$ & 0.005 & 1.45 & $\begin{array}{l}(1.02- \\
2.07)\end{array}$ & 0.041 & 0.002 & 1.41 & $\begin{array}{l}(1.03- \\
1.92)\end{array}$ & 0.033 \\
\hline $\begin{array}{l}\text { Operative type } \\
\text { (lap vs open) }\end{array}$ & 0.014 & 1.81 & $\begin{array}{l}(1.24- \\
2.65)\end{array}$ & 0.002 & 0.014 & 1.55 & $\begin{array}{l}(1.12- \\
2.13)\end{array}$ & 0.008 \\
\hline \multicolumn{9}{|c|}{$\begin{array}{l}\text { PSM, propensity score matching; OS, overall survival; RFS, recurrent-free survival; } \mathrm{Cl} \text {, confidence } \\
\text { interval; HBV, hepatitis B virus; WBC, white blood cells; HGB, hemoglobin; PT, prothrombin time; PLT, } \\
\text { platelets; ALB, albumin; AST, aspartate aminotransferase; Glu, glucose; Cr, creatinine; TBIL, serum total } \\
\text { bilirubin; AFP, alpha-fetoprotein; MVI, microvascular invasion }\end{array}$} \\
\hline
\end{tabular}

\section{Intraoperative And Postoperative Parameters Of Hcc Patients}

The median operative time ( $160 \mathrm{~min}$ vs $180 \mathrm{~min}, \mathrm{P}<0.05)$, median blood loss ( $200 \mathrm{ml}$ vs $300 \mathrm{ml}, \mathrm{P}<0.001)$ and the frequency of blood transfusion $(8.64 \%$ vs $20.2 \%, P<0.001)$ were significantly lower in the laparoscopic group than the laparotomic group (Table S1). However, the laparoscopic hepatectomy was more likely to require the vascular inflow occlusion (Pringle's maneuver) ( $30 \%$ vs $17.5 \%, P=0.001)$ compared with the laparotomic liver resection. The postoperative complication rates were analyzed as well. As shown in Table 2, the rates of hydrothorax and ascites were significantly lower in the laparoscopic group than the laparotomic group $(2.06 \%$ vs $7.14 \%, P<0.05 ; 2.06 \%$ vs $10.3 \%, P<0.001$, respectively). The rates of other complications, such as hepatic failure and bile leakage, were comparable in the two groups.

\section{Discussion}


In this study, the PSM analysis was used to construct a matched group of HCC patients for comparison of follow-up indicators including laboratory examination and operation features between the laparoscopic and open hepatectomy groups to minimize selection biases. The results showed that the OS and RFS of patients without MVI were significantly improved in the laparoscopic group than the laparotomic group before and after PSM. The OS and RFS of patients with MVI were also remarkably increased in the laparoscopic group compared with the laparotomic group before PSM; and the OS and RFS of patients with MVI were equivalent between the laparoscopic and laparotomic groups after PSM. Thus, laparoscopic hepatectomy can be considered as a feasible alternative in HCC patients at BCLC stages 0 or A regardless of the presence of MVI.

Also, operative outcomes of laparoscopy and laparotomy were analyzed in this study. It was shown that laparoscopic hepatectomy had a shorter operative time, less blood loss and lower frequency of blood transfusion; and the recurrence and mortality rates were also decreased in the laparoscopic group. We speculated the higher efficacy and less need for blood transfusion of laparoscopic hepatectomy may be associated with the small incision and less surgical trauma. A previous study demonstrated that laparoscopic right hepatectomy was associated with a tendency of prolonged operative time and less intraoperative blood loss after PSM[24]. We speculated that the prolonged operative time may be related to the relatively difficult operation technique and the limited vision field of the right hepatectomy. Toru et al.[25] reported that the median estimated blood loss and median postoperative hospital stay were significantly reduced in the laparoscopic group, but the laparoscopic and laparotomic groups had similar morbidity and mortality rates. Additionally, the postoperative complication rates were also analyzed in the present study. We found that the rates of hydrothorax and ascites were significantly lower in the laparoscopic group compared to the laparotomic group, because the laparoscopy is less traumatic than laparotomy. However, a previous study reported that postoperative complication rates were comparable between the two groups in the PSM cohort[25]. Hence, the operative time, the safety degree and prognosis of these two operation approaches are still controversial. The different outcomes in these studies can be affected by the skill and experience of operators.

Burgeoning literature compared the impact of laparoscopic and laparotomic hepatectomy on patients' survival. A meta-analysis in 2013 including 1238 patients from 15 studies indicated that the laparoscopic group may have short-term advantages in terms of blood loss and postoperative morbidity for HCC, but both procedures have similar long-term outcomes[26]. Another meta-analysis in 2018 showed that laparoscopic hepatectomy was associated with significantly lower tumor recurrence; the laparoscopic group conferred greater overall survival at 1- and 5-years [27]. No significant difference was found in longterm outcomes between the laparoscopic and laparotomic groups in early years, and the possible reasons caused this phenomenon may be as follows: (1) Laparoscopic technology was not mature at that time; (2) The indication of laparoscopy was primarily limited to minor hepatectomy; few major hepatectomy operations were performed using laparoscopy; (3) No advanced assisting technologies, such as intraoperative ultrasound and immunofluorescence, were applied during operations. 
The presence of MVI worsens survival outcomes of HCC postoperatively, an accurate preoperative prediction of MVI can help surgeons to better choose surgical procedures[28]. Nevertheless, it is elusive whether MVI can provide prognostic information for patients at a specific tumor stage. Many efforts have been made to preoperatively estimate the presence of MVI. Some studies reported that some types of MVI are shown as the "typical dynamical pattern" on contrast-enhanced MRI[29, 30]; while other studies recommended the detection of serum tumor biomarkers to evaluate MVI[31]. However, the clinical practicability of these methods in the preoperative risk estimation of MVI remains to be confirmed.

Cheng et al.[7] documented that the occurrence rates of MVI at BCLC stages $0, A$, and B were $12.4 \%$, $26.2 \%$, and $34.4 \%$, respectively. Besides, they found MVI was an independent risk factor for OS and RFS in patients at BCLC stage A. Wang et al[32]. showed MVI could predict an adverse recurrence pattern and poor prognosis and had the potential to be used as a reference index when deciding whether to operate. In line with the previous studies, we also confirmed worse survival outcomes in HCC patients with MVI.

Besides, our study demonstrated that the long-term prognosis of patients without MVI who underwent laparoscopic recection were significantly better than that of patients without MVI who received open resection before and after PSM. We speculated that loss of blood, length of hospitalization and postoperative complications were potential factors which influenced patients' survival [33-36]. On the other hand, patients diagnosed with MVI who underwent laparoscopic recection had similar long-term survival compared with patients associated with MVI who were treated with open resection before and after PSM. We hypothesized that the survival benefits of patients who underwent laparoscopic recection were counteracted by the presence of MVI. Thus, from a conservative viewpoint, our results can be interpreted to indicate that laparoscopy is at least not inferior to the standard open resection approach for HCC patients at BCLC stages 0 or A associated with MVI or not.

The present study has several limitations. First, this is a retrospective study with its inherent selection bias. Further prospective research needs to be designed and conducted. Next, although the number of patients enrolled in our study is relatively large, the sample size is still insufficient to draw a firm conclusion about the effect of MVI on the surgical choice in HCC patients. Last, this study was conducted in China with most patients having a background of HBV infection, whether the results of this study can be extrapolated to other different races and etiologies requires more studies.

\section{Conclusion}

Taken together, our study elucidated that for HCC patients at BCLC stages 0 or A, the long-term outcomes of patients who were treated with laparoscopic approach were at least not inferior to those of patients who underwent laparotomy approach Therefore, laparoscopic liver resection may be a safe and feasible alternative for $\mathrm{HCC}$ patients at early stages, regardless of the presence of MVI.

\section{Declarations}


The data that support the findings of this study are available from the corresponding author upon reasonable request.

\section{Animal Research (Ethics)}

Not applicable.

\section{Ethics Approval and Consent to Participate (Ethics)}

All procedures performed in this study involving human participants were approved by the Institutional Ethics Review Board of the Eastern Hepatobiliary Surgery Hospital, Fujian Provincial Hospital, The Third Affiliated Hospital of Soochow University, and Affiliated Tumor Hospital of Guangxi Medical University. This study was in accordance with the 1964 Helsinki declaration and its later amendments or comparable ethical standards. Written informed consent for study participation was obtained from all the patients.

\section{Consent to Publish (Ethics)}

All authors confirm that the work described has not been published before and is not under consideration for publication elsewhere. All authors have seen and gave consent to the publication of this study. The publication of this work has been approved by the responsible authorities at the institution where the work is carried out.

\section{Clinical Trials Registration}

Not applicable because this is a retrospective observational study.

\section{Conflict of Interest:}

The authors declare that they have no competing financial interests or personal relationships that could have appeared to influence the work reported in this paper.

Funding: Meng Chao Talent Training Program (EHBH2019YC112); National Natural Science Foundation of China (82172846)

\section{Author Contribution:}

Conception and design: Wei-Xing Guo, Shu-Qun Cheng, Shi-Ye Yang, Mao-Lin Yan, Yun-Fei Duan, Jin-Kai Feng, Jia-Zhou Ye;

Administrative support: Wei-Xing Guo, Shu-Qun Cheng;

Provision of study materials or patients: Wei-Xing Guo, Shu-Qun Cheng, Mao-Lin Yan, Yun-fei Duan, JiaZhou Ye;

Collection and assembly of data: Shi-Ye Yang, Lei Guo, Jie Xue; 
Data analysis and interpretation: Shi-Ye Yang, Jin-Kai Feng, Yan-Jun Xiang, Zong-Han Liu

Statistical analysis: Shi-Ye Yang, Jin-Kai Feng, Yan-Jun Xiang, Zong-Han Liu

Manuscript writing: All authors;

Final approval of manuscript: All authors.

\section{Synopsis:}

For hepatocellular carcinoma (HCC) patients at BCLC stage 0-A with or without microvascular invasion $(\mathrm{MVI})$, laparoscopic liver resection may be a safe and feasible alternative for laparotomic liver resection.

\section{Acknowledgment}

This study was supported by Meng Chao Talent Training Program (EHBH2019YC112) and National Natural Science Foundation of China (82172846)

\section{References}

1. Sarveazad A, Agah S, Babahajian A, Amini N, Bahardoust M. Predictors of 5 year survival rate in hepatocellular carcinoma patients. J Res Med sciences: official J Isfahan Univ Med Sci. 2019;24:86.

2. Bertuccio P, Turati F, Carioli G, Rodriguez T, La Vecchia C, Malvezzi M, Negri E. Global trends and predictions in hepatocellular carcinoma mortality. J Hepatol. 2017;67(2):302-9.

3. Lei Z, Li J, Wu D, Xia Y, Wang Q, Si A, Wang K, Wan X, Lau WY, Wu M, et al. Nomogram for Preoperative Estimation of Microvascular Invasion Risk in Hepatitis B Virus-Related Hepatocellular Carcinoma Within the Milan Criteria. JAMA Surg. 2016;151(4):356-63.

4. Bai S, Yang P, Xie Z, Li J, Lei Z, Xia Y, Qian G, Zhang B, Pawlik TM, Lau WY, et al. Preoperative Estimated Risk of Microvascular Invasion is Associated with Prognostic Differences Following Liver Resection Versus Radiofrequency Ablation for Early Hepatitis B Virus-Related Hepatocellular Carcinoma. Ann Surg Oncol. 2021;28(13):8174-85.

5. Imai K, Yamashita YI, Yusa T, Nakao Y, Itoyama R, Nakagawa S, Okabe H, Chikamoto A, Ishiko T, Baba H. Microvascular Invasion in Small-sized Hepatocellular Carcinoma: Significance for Outcomes Following Hepatectomy and Radiofrequency Ablation. Anticancer Res. 2018;38(2):1053-60.

6. Lee S, Kang TW, Song KD, Lee MW, Rhim H, Lim HK, Kim SY, Sinn DH, Kim JM, Kim K, et al. Effect of Microvascular Invasion Risk on Early Recurrence of Hepatocellular Carcinoma After Surgery and Radiofrequency Ablation. Ann Surg. 2021;273(3):564-71.

7. Huang C, Zhu XD, Ji Y, Ding GY, Shi GM, Shen YH, Zhou J, Fan J, Sun HC. Microvascular invasion has limited clinical values in hepatocellular carcinoma patients at Barcelona Clinic Liver Cancer (BCLC) stages 0 or B. BMC Cancer. 2017;17(1):58. 
8. Xiang Y-J, Wang K, Zheng Y-T, Yu H-M, Cheng Y-Q, Wang W-J, Shan Y-F, Cheng S-Q. Prognostic Value of Microvascular Invasion in Eight Existing Staging Systems for Hepatocellular Carcinoma: A BiCenteric Retrospective Cohort Study. Frontiers in Oncology 2021, 11(5357).

9. Chen ZH, Zhang XP, Feng JK, Li LQ, Zhang F, Hu YR, Zhong CQ, Shi J, Guo WX, Wu MC, et al. Actual long-term survival in hepatocellular carcinoma patients with microvascular invasion: a multicenter study from China. Hepatol Int. 2021;15(3):642-50.

10. Okamura Y, Sugiura T, Ito T, Yamamoto Y, Ashida R, Ohgi K, Aramaki T, Uesaka K. Anatomical resection is useful for the treatment of primary solitary hepatocellular carcinoma with predicted microscopic vessel invasion and/or intrahepatic metastasis. Surg Today. 2021;51(9):1429-39.

11. Sun Z, Li Z, Shi XL, He XW, Chen J, Song JH. Anatomic versus non-anatomic resection of hepatocellular carcinoma with microvascular invasion: A systematic review and meta-analysis. Asian J Surg. 2021;44(9):1143-50.

12. Zhao H, Chen C, Gu S, Yan X, Jia W, Mao L, Qiu Y. Anatomical versus non-anatomical resection for solitary hepatocellular carcinoma without macroscopic vascular invasion: A propensity score matching analysis. J Gastroenterol Hepatol. 2017;32(4):870-8.

13. Hu H, Qi S, Zeng S, Zhang P, He L, Wen S, Zeng N, Yang J, Zhang W, Zhu W, et al. Importance of Microvascular Invasion Risk and Tumor Size on Recurrence and Survival of Hepatocellular Carcinoma After Anatomical Resection and Non-anatomical Resection. Front Oncol. 2021;11:621622.

14. Yoshida H, Taniai N, Yoshioka M, Hirakata A, Kawano Y, Shimizu T, Ueda J, Takata H, Nakamura Y, Mamada Y. Current Status of Laparoscopic Hepatectomy. J Nippon Med Sch. 2019;86(4):201-6.

15. Twaij A, Pucher PH, Sodergren MH, Gall T, Darzi A, Jiao LR: Laparoscopic vs open approach to resection of hepatocellular carcinoma in patients with known cirrhosis: systematic review and metaanalysis. World journal of gastroenterology 2014, 20(25):8274-8281.

16. Zhou YM, Shao WY, Zhao YF, Xu DH, Li B. Meta-analysis of laparoscopic versus open resection for hepatocellular carcinoma. Dig Dis Sci. 2011;56(7):1937-43.

17. Sotiropoulos GC, Prodromidou A, Kostakis ID, Machairas N. Meta-analysis of laparoscopic vs open liver resection for hepatocellular carcinoma. Updates Surg. 2017;69(3):291-311.

18. Asrani SK, Ghabril MS, Kuo A, Merriman RB, Morgan T, Parikh ND, Ovchinsky N, Kanwal F, Volk ML, Ho C, et al: Quality measures in HCC care by the Practice Metrics Committee of the American Association for the Study of Liver Diseases. Hepatology (Baltimore, Md) 2021.

19. Adhoute X, Penaranda G, Raoul JL, Bourlière M. Nomogram of the Barcelona Clinic Liver Cancer System: external validation in European patients. Liver international: official journal of the International Association for the Study of the Liver. 2016;36(11):1716-7.

20. Zhang EL, Cheng Q, Huang ZY, Dong W. Revisiting Surgical Strategies for Hepatocellular Carcinoma With Microvascular Invasion. Front Oncol. 2021;11:691354.

21. Li B, Liu T, Zhang Y, Zhang J. Retroperitoneal laparoscopic hepatectomy of recurrent hepatocellular carcinoma: case report and literature review. BMC Gastroenterol. 2020;20(1):278. 
22. Abu Hilal M, Aldrighetti L, Dagher I, Edwin B, Troisi RI, Alikhanov R, Aroori S, Belli G, Besselink M, Briceno J, et al. The Southampton Consensus Guidelines for Laparoscopic Liver Surgery: From Indication to Implementation. Ann Surg. 2018;268(1):11-8.

23. Han HS, Shehta A, Ahn S, Yoon YS, Cho JY, Choi Y. Laparoscopic versus open liver resection for hepatocellular carcinoma: Case-matched study with propensity score matching. J Hepatol. 2015;63(3):643-50.

24. Chen K, Pan Y, Wang YF, Zheng XY, Liang X, Yu H, Cai XJ. Laparoscopic Right Hepatectomy for Hepatocellular Carcinoma: A Propensity Score Matching Analysis of Outcomes Compared with Conventional Open Surgery. J laparoendoscopic Adv Surg techniques Part A. 2019;29(4):503-12.

25. Beppu T, Wakabayashi G, Hasegawa K, Gotohda N, Mizuguchi T, Takahashi Y, Hirokawa F, Taniai N, Watanabe $\mathrm{M}$, Katou $\mathrm{M}$, et al. Long-term and perioperative outcomes of laparoscopic versus open liver resection for colorectal liver metastases with propensity score matching: a multi-institutional Japanese study. J Hepato-Biliary-Pancreat Sci. 2015;22(10):711-20.

26. Yin Z, Fan X, Ye H, Yin D, Wang J: Short- and long-term outcomes after laparoscopic and open hepatectomy for hepatocellular carcinoma: a global systematic review and meta-analysis. Annals of surgical oncology 2013, 20(4):1203-1215.

27. Goh EL, Chidambaram S, Ma S. Laparoscopic vs open hepatectomy for hepatocellular carcinoma in patients with cirrhosis: A meta-analysis of the long-term survival outcomes. Int J Surg (London England). 2018;50:35-42.

28. Bai S, Yang P, Xie Z, Li J, Lei Z, Xia Y, Qian G, Zhang B, Pawlik TM, Lau WY, et al: Preoperative Estimated Risk of Microvascular Invasion is Associated with Prognostic Differences Following Liver Resection Versus Radiofrequency Ablation for Early Hepatitis B Virus-Related Hepatocellular Carcinoma. Annals of surgical oncology 2021.

29. Witjes CD, Willemssen FE, Verheij J, van der Veer SJ, Hansen BE, Verhoef C, de Man RA, ljzermans JN. Histological differentiation grade and microvascular invasion of hepatocellular carcinoma predicted by dynamic contrast-enhanced MRI. J Magn Reson imaging: JMRI. 2012;36(3):641-7.

30. Choi YS, Rhee H, Choi JY, Chung YE, Park YN, Kim KW, Kim MJ. Histological characteristics of small hepatocellular carcinomas showing atypical enhancement patterns on gadoxetic acid-enhanced MR imaging. J Magn Reson imaging: JMRI. 2013;37(6):1384-91.

31. Gouw AS, Balabaud C, Kusano H, Todo S, Ichida T, Kojiro M: Markers for microvascular invasion in hepatocellular carcinoma: where do we stand? Liver transplantation: official publication of the American Association for the Study of Liver Diseases and the International Liver Transplantation Society 2011, 17 Suppl 2:S72-80.

32. Wang H, Qian YW, Wu MC, Cong WM. Liver Resection Is Justified in Patients with BCLC Intermediate Stage Hepatocellular Carcinoma without Microvascular Invasion. J Gastrointest surgery: official J Soc Surg Aliment Tract. 2020;24(12):2737-47.

33. Ivanics T, Claasen MP, Patel MS, Rajendran L, Shwaartz C, Raschzok N, et al: Long-term outcomes of laparoscopic liver resection for hepatocellular carcinoma: A propensity score matched analysis of a 
high-volume North American center. Surgery 2021, S0039-6060(21)00977-6.

34. Meng X, Xu Y, Pan Y, Lu S, Duan W. Open versus laparoscopic hepatic resection for hepatocellular carcinoma: a systematic review and meta-analysis. Surg Endosc. 2019;33(8):2396-418.

35. Cheung TT, Dai WC, Tsang SH, Chan AC, Chok KS, Chan SC, et al. Pure Laparoscopic Hepatectomy Versus Open Hepatectomy for Hepatocellular Carcinoma in 110 Patients With Liver Cirrhosis A Propensity Analysis at a Single Center. Ann Surg. 2016;264(4):612-20.

36. Katz SC, Shia J, Liau KH, Gonen M, Ruo L, Jarnagin WR, et al. Operative Blood Loss Independently Predicts Recurrence and Survival After Resection of Hepatocellular Carcinoma. Ann Surg. 2009;249(4):617-23.

\section{Figures}




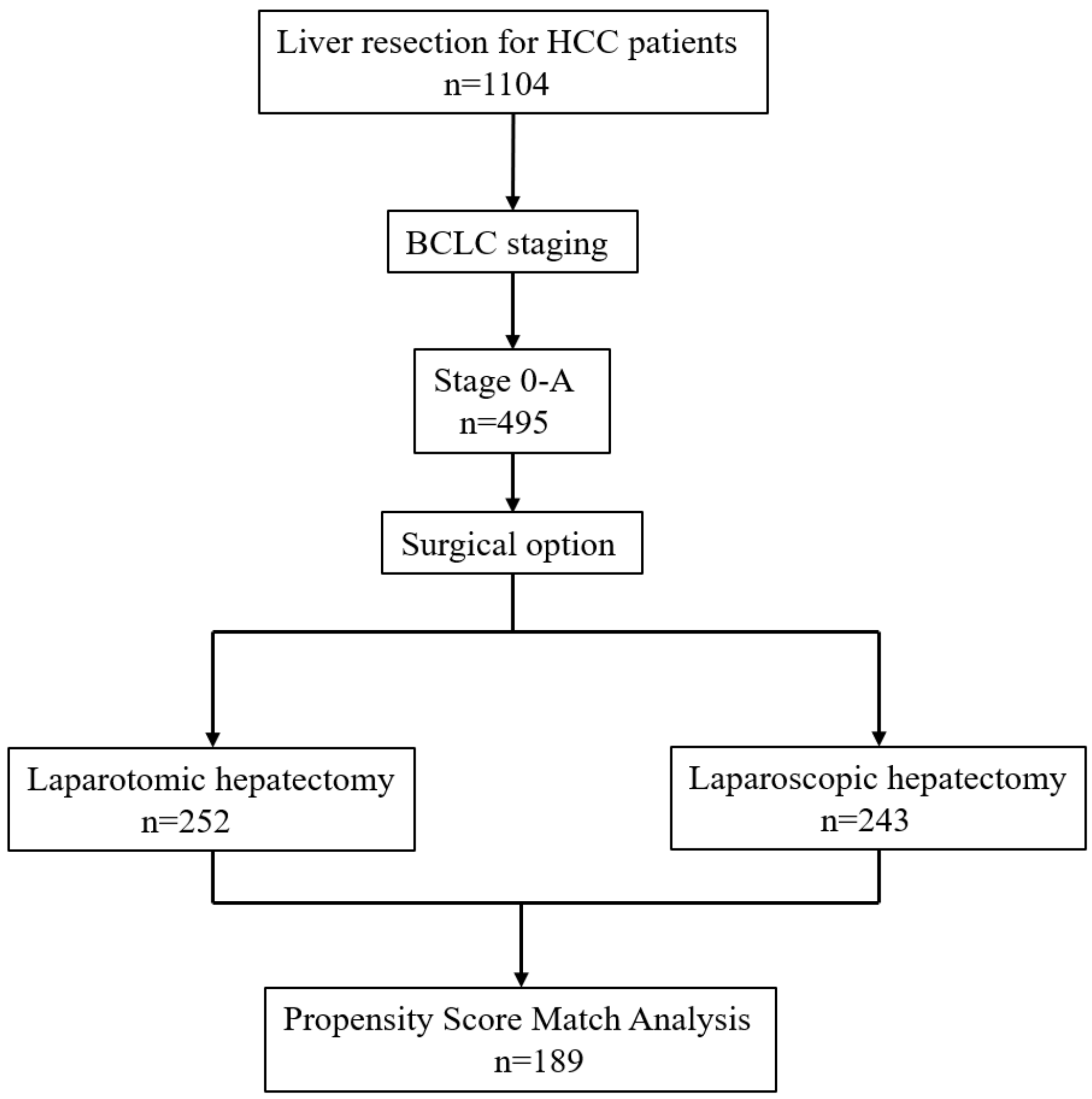

Figure 1

Study design frame, patient selection, and propensity score matching. HCC, hepatocellular carcinoma; BCLC, Barcelona Clinic Liver Cancer

Figure 2 
Overall survival (OS) and recurrence-free survival (RFS) of BCLC stages 0-A HCC patients treated with laparoscopy or laparotomy before and after PSM. OS (A) and RFS (B) of HCC patients at BCLC stages 0A before PSM. OS (C) and RFS (D) of HCC patients at BCLC stages $0-A$ after PSM.

\section{Figure 3}

Overall survival (OS) and recurrence-free survival (RFS) of BCLC stages 0-A HCC patients with or without microvascular invasion (MVI) treated with laparoscopy or laparotomy before PSM. OS (A) and RFS (B) of HCC patients at BCLC stages $0-A$ without MVI before PSM. OS (C) and RFS (D) of HCC patients at BCLC stages $0-A$ with MVI before PSM.

\section{Figure 4}

Overall survival (OS) and recurrence-free survival (RFS) of BCLC stages 0-A HCC patients with or without microvascular invasion (MVI) treated with laparoscopy or laparotomy after PSM. OS (A) and RFS (B) of HCC patients at BCLC stages 0-A without MVI after PSM. OS (C) and RFS (D) of HCC patients at BCLC stages $0-A$ with MVI after PSM.

\section{Supplementary Files}

This is a list of supplementary files associated with this preprint. Click to download.

- SupplementaryTable1.docx 